\title{
DEL PEZZO SURFACES OF DEGREE 6
}

\author{
PATRICK CORN
}

\begin{abstract}
We give a correspondence which associates, to each Del Pezzo surface $X$ of degree 6 over a field $k$ of characteristic 0 , a collection of data consisting of a Severi-Brauer variety/ $k$ and a set of points defined over some extension of $k$.
\end{abstract}

The main results in this paper, and specifically Theorem 5.1, give a way to describe Del Pezzo surfaces of degree 6 over a field $k$ of characteristic 0 , via a correspondence with objects (Severi-Brauer varieties) which can be understood in a completely explicit way if $k$ is sufficiently nice (e.g. $k$ a number field).

\section{Preliminaries}

In this paper, we will deal with varieties $V$ over a field $k$ of characteristic 0 . If $L / k$ is a field extension, then we write $V_{L}$ for the base extension $V \times{ }_{\operatorname{Spec} k} \operatorname{Spec} L$, and $\bar{V}$ for $V_{\bar{k}}$.

A Del Pezzo surface over a number field $k$ is a smooth rational surface $X$ whose anticanonical sheaf $\omega_{X}^{-1}$ is ample. To each Del Pezzo surface $X$ is associated a number $d=\left(\omega_{X}, \omega_{X}\right)$ (where (, ) denotes intersection number), called the degree of $X$.

The results we need about Del Pezzo surfaces are summarized in the following proposition. We refer the interested reader to [Man74] for proofs and more details.

Proposition 1.1. Let $V$ be a Del Pezzo surface of degree $d$ over a field $k$.

(a) $1 \leq d \leq 9$.

(b) Pic $\bar{V}$ is a free abelian group of rank $10-d$.

(c) If $V^{\prime} \rightarrow V$ is a birational morphism and $V^{\prime}$ is a Del Pezzo surface, then $V$ is a Del Pezzo surface.

(d) Either $\bar{V}$ is isomorphic to the blowup of $\mathbb{P}_{k}^{2}$ at $r=9-d$ points $\left\{x_{1}, \ldots, x_{r}\right\}$ in general position, or $d=8$ and $\bar{V} \cong \mathbb{P}_{\bar{k}} \times \mathbb{P}_{\bar{k}}$. Conversely, if $r \leq 6$, then any surface satisfying this condition is a Del Pezzo surface of degree $d=9-r$. (For a set of $r \leq 6$ points, "general position" means that no three are collinear and no six lie on a conic.)

(e) Suppose $f: \bar{V} \rightarrow \mathbb{P}_{\bar{k}}^{2}$ is a map which expresses $\bar{V}$ as the blowup of $\mathbb{P}_{\bar{k}}^{2}$ at $r$ points $\left\{x_{1}, \ldots, x_{r}\right\}$ in general position. Let $C$ be an exceptional curve; that is, $C$ is a curve on $\bar{V}$ such that $(C, C)=-1$ and $C \cong \mathbb{P}_{\bar{k}}$. Then if $r \leq 6, f(C)$ is either: one of the $x_{i}$, a line passing through two of the $x_{i}$, or a conic passing

Received August 3, 2004. 
through five of the $x_{i}$. Conversely, each point, line, and conic in this list gives rise to exactly one exceptional curve $C$.

Proof. These are (respectively) Theorem 24.3(i), Lemma 24.3.1, Corollary 24.5.2, Theorem 24, and Theorem 26.2 of [Man74].

The assumption that $r \leq 6$ was made only to simplify the statements of (d) and (e); we will not be concerned with Del Pezzo surfaces of degree 1 or 2 in this paper.

\section{Severi-Brauer varieties: the basic construction}

If $V$ is a Del Pezzo surface over a field $k$, it is clear from the definition above that the exceptional curves on $\bar{V}$ are preserved by the action of $G_{k}:=\operatorname{Gal}(\bar{k} / k)$; this information can be very useful in investigating properties of these surfaces.

Now let $D$ be a Del Pezzo surface of degree 6 . Let $E_{1}, E_{2}$, and $E_{3}$ be the exceptional curves corresponding to the blow-ups of the three points $x_{1}, x_{2}, x_{3} \in$ $\mathbb{P}_{\bar{k}}^{2}$ as in Proposition 1.1(d). Let $F_{12}$ be the exceptional curve corresponding to the line between $x_{1}$ and $x_{2}$, and define $F_{13}$ and $F_{23}$ similarly. By Proposition 1.1(e), the set $\left\{E_{1}, E_{2}, E_{3}, F_{12}, F_{13}, F_{23}\right\}$ is precisely the set of exceptional curves on $D$.

We can now examine the possibilities for blowing down these curves to obtain other, possibly simpler surfaces.

Proposition 2.1. Let $D$ be a Del Pezzo surface of degree six over a field $k$ of characteristic 0 . There is a field $L$ such that $[L: k]=1$ or 2 and surfaces $X$ and $Y$ defined over $L$ such that the triple $\left(D_{L}, X, Y\right)$ satisfies the following conditions:

(i) there is a morphism $\pi_{X}: D_{L} \rightarrow X$ which exhibits $D_{L}$ as the blow-up of $X$ at a $G_{L}$-stable set of three non-collinear points $\left\{P_{1}, P_{2}, P_{3}\right\} \in X(\bar{k})$

(ii) there is a morphism $\pi_{Y}: D_{L} \rightarrow Y$ which exhibits $D_{L}$ as the blow-up of $Y$ at a $G_{L}$-stable set of three non-collinear points $\left\{Q_{1}, Q_{2}, Q_{3}\right\} \in Y(\bar{k})$

(iii) $\left\{\pi_{X}^{-1}\left(P_{i}\right): 1 \leq i \leq 3\right\} \cup\left\{\pi_{Y}^{-1}\left(Q_{i}\right): 1 \leq i \leq 3\right\}$ is a full set of six exceptional curves on $\bar{D}$.

(iv) $X$ and $Y$ are Severi-Brauer varieties of dimension 2.

Proof. Let $L$ be the minimal field such that the sets

$$
\left\{E_{1}, E_{2}, E_{3}\right\} \text { and }\left\{F_{12}, F_{13}, F_{23}\right\}
$$

are both $G_{L^{-}}$-stable. Any element of $G_{k}$ either fixes both sets or switches them, so $L$ is either equal to $k$ or quadratic over $k$. Let $X^{\prime}$ and $Y^{\prime}$ be the varieties obtained from blowing down $\left\{E_{1}, E_{2}, E_{3}\right\}$ and $\left\{F_{12}, F_{13}, F_{23}\right\}$, respectively, over $\bar{k}$. Then they can naturally be descended to varieties $X$ and $Y$ defined over $L$ (see [Wei56] for details on descent). Properties (i)-(iii) are immediate.

To see property (iv), note that $X$ and $Y$ are Del Pezzo surfaces by Proposition 1.1(c). Now note that rank Pic $\bar{D}=4$ by Proposition 1.1(b), and blowing up at a point increases the rank of the Picard group by 1 , so rank Pic $\bar{X}$ must be 1 . 
Then by Proposition 1.1(b) the degree of $X$ is 9 , which means $r=0$, so $X$ is a twist of $\mathbb{P}^{2}$. The same holds for $Y$.

Now we can also turn Proposition 2.1 around:

Proposition 2.2. Let $X$ be a Severi-Brauer variety over a field $L$ and let $\left\{P_{1}, P_{2}, P_{3}\right\}$ be a $G_{L}$-stable set of non-collinear points in $X(\bar{L})$. Then there exist $S$ and $Y$ defined over $L$ such that the triple $(S, X, Y)$ satisfies conditions (i)-(iv) of Proposition 2.1.

Proof. To obtain $S$, simply blow up $X$ over $L$ at the given set of points. To obtain $Y$, note that the three exceptional curves on $\bar{S}$ which are the inverse images of $\left\{P_{1}, P_{2}, P_{3}\right\}$ form a $G_{L}$-stable set (call it $C_{1}$ ), and since the full set $C$ of exceptional curves is $G_{L}$-stable, the complement $C \backslash C_{1}$ is also $G_{L}$-stable and can be blown down over $L$ to obtain $Y$. Conditions (i)-(iii) are all obvious from the construction.

There is a natural one-to-one correspondence between (isomorphism classes of) Severi-Brauer varieties over $L$ of dimension 2 and (isomorphism classes of) central simple algebras over $L$ of dimension 9 ; both are parametrized by $H^{1}\left(G_{L}, P G L_{3}(\bar{L})\right)$. (Cf. [Ser79].)

Given the result of proposition 2.2, the natural question to ask is: how are the central simple algebras corresponding to $X$ and $Y$ related? The answer is our first main result.

Theorem 2.3. Let $X$ be a Severi-Brauer variety of dimension 2 over a field $L$, equipped with a $G_{L}$-stable set $\left\{P_{1}, P_{2}, P_{3}\right\}$ of three non-collinear points. Construct $S$ and $Y$ as in Proposition 2.1. Let $x$ and $y$ be the central simple algebras corresponding to $X$ and $Y$ respectively. Then $y=x^{\mathrm{op}}$, the opposite algebra of $x$.

Proof. We simply unravel the definition of the correspondence between SeveriBrauer varieties and central simple algebras. First, choose an ordering of the points $\left\{P_{1}, P_{2}, P_{3}\right\}$ and the points $\left\{Q_{1}, Q_{2}, Q_{3}\right\}$ of proposition 2 so that $\pi_{X}^{-1}\left(P_{i}\right) \cap$ $\pi_{Y}^{-1}\left(Q_{i}\right)=\emptyset$ for all $i$.

Let $M$ be the minimal Galois extension of $L$ over which the $P_{i}$ are each individually defined. Then the $Q_{i}$ are all defined over $M$ as well. Also, $S_{M}$ is isomorphic to the blowup of $\mathbb{P}_{M}^{2}$ at the $P_{i}$, so we can choose a point $P \in S(M)$ which lies over a point in $\mathbb{P}^{2}(M) \backslash\left\{P_{1}, P_{2}, P_{3}\right\}$, so that $P$ does not lie on any exceptional curve. Now $X_{M} \cong \mathbb{P}_{M}^{2}$, and since the automorphism group of $\mathbb{P}_{M}^{2}$ acts transitively on sets of four $M$-points in general position, we can construct an isomorphism $\phi: X_{M} \rightarrow \mathbb{P}_{M}^{2}$ sending the points $P_{1}, P_{2}, P_{3},\left(\pi_{X}\right)_{M}(P)$ on $X$ (notation as in Proposition 2.1) to $(1: 0: 0),(0: 1: 0),(0: 0: 1)$, and $(1: 1: 1)$, respectively. We can also construct an isomorphism $\psi: Y_{M} \rightarrow \mathbb{P}_{M}^{2}$ sending $Q_{1}, Q_{2}, Q_{3},\left(\pi_{Y}\right)_{M}(P)$ on $Y$ (notation as in Proposition 2.1) to $(1: 0: 0),(0: 1:$ $0),(0: 0: 1)$, and $(1: 1: 1)$, respectively. 


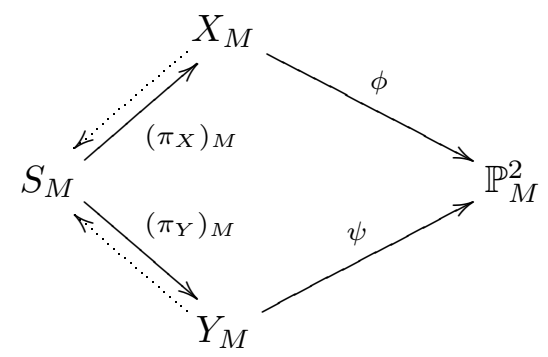

Starting at $\mathbb{P}_{M}^{2}$ and going around counterclockwise in the diagram, we have a rational map $b:=\psi \circ\left(\pi_{Y}\right)_{M} \circ\left(\pi_{X}\right)_{M}^{-1} \circ \phi^{-1}$. We can easily write down a formula for this map, as in [Har77], pp. 397-398:

$$
b(x: y: z)=(y z: x z: x y) .
$$

So $b$ is invariant under the natural action of $G_{M / L}:=\operatorname{Gal}(M / L)$.

But the composition $d:=\left(\pi_{Y}\right)_{M} \circ\left(\pi_{X}\right)_{M}^{-1}$ is also $G_{M / L}$-invariant. And $b=$ $\psi \circ d \circ \phi^{-1}$, so

$$
d=\psi^{-1} \circ b \circ \phi
$$

Now take $\sigma \in G_{M / L}$. Since ${ }^{\sigma} d=d$ and ${ }^{\sigma} b=b$, we have

$$
\begin{aligned}
\sigma\left(\psi^{-1} b \phi\right) & =\psi^{-1} b \phi \\
\psi\left({ }^{\sigma} \psi^{-1}\right) & =b \phi\left({ }^{\sigma} \phi^{-1}\right) b^{-1}
\end{aligned}
$$

Recall that the correspondence between Severi-Brauer varieties with points in $M$ and central simple algebras split by $M$ is via the cohomology group $H^{1}\left(G_{M / L}, P G L_{3}(M)\right)$. The cocycles associated to $X$ and $Y$ are precisely $\eta_{\sigma}:=$ $\phi\left({ }^{\sigma} \phi^{-1}\right)$ and $\xi_{\sigma}:=\psi\left({ }^{\sigma} \psi^{-1}\right)$. So (1) translates to:

$$
\xi_{\sigma}=b \eta_{\sigma} b^{-1} \text {. }
$$

Now, for any $\sigma \in G_{M / L}$, the set $\{(1: 0: 0),(0: 1: 0),(0: 0: 1)\}$ is stable under $\eta_{\sigma}$ and $\xi_{\sigma}$, considered as automorphisms of $\mathbb{P}^{2}$. So $\eta_{\sigma}$ and $\xi_{\sigma}$ land in the subgroup

$H:=\left\{A \in P G L_{3}(M)\right.$ : each row and column of $A$ has exactly one nonzero entry $\}$.

(For simplicity, we often abuse notation and identify elements of $P G L_{3}(M)$ with representative matrices in $G L_{3}(M)$.)

Clearly $H$ decomposes as a semi-direct product

$$
H=H_{D} \rtimes H_{P},
$$

where $H_{D}$ is the subgroup of diagonal matrices and $H_{P}$ the subgroup of permutation matrices in $P G L_{3}(M)$. In particular, any matrix in $H$ can be written $A=A_{D} A_{P}$, where $A_{D}$ is diagonal and $A_{P}$ is a permutation matrix.

Conjugation by $b$ sends $A_{D}$ to $A_{D}^{-1}$, and sends $A_{P}$ to $A_{P}$. Note that

$$
b A_{D} A_{P} b^{-1}=A_{D}^{-1} A_{P}=\left({ }^{t} A_{D}^{-1}\right)\left({ }^{t} A_{P}^{-1}\right),
$$


or, in other words, conjugation by $b$ is the same as applying the operator $A \mapsto$ ${ }^{t} A^{-1}$ on $H$.

Next we prove a lemma about this operator.

Lemma 2.4. Let $c: G_{M / L} \rightarrow P G L_{3}(M)$ be a cocycle corresponding to a central simple algebra $x$. Precomposing $c$ with the map $A \mapsto{ }^{t} A^{-1}$ on $P G L_{3}(M)$ sends the class of $x$ to the inverse of the class of $x$ in $\operatorname{Br}(L)$.

Proof of lemma. By definition of the correspondence between $c$ and $x, c$ is constructed by the following formula: there is some isomorphism $\alpha: x \otimes_{k} \bar{k} \rightarrow M_{3}(\bar{k})$ such that for any $\sigma \in G_{M / L}$ and $A \in M_{3}(\bar{k})$,

$$
c_{\sigma} A c_{\sigma}^{-1}=\alpha\left({ }^{\sigma} \alpha^{-1}\right)(A) .
$$

If we let $\beta(A)={ }^{t} \alpha(A)$, then $\beta$ is an isomorphism $x^{\mathrm{op}} \otimes \bar{k} \rightarrow M_{3}(\bar{k})$. And

$$
\beta\left({ }^{\sigma} \beta^{-1}\right)(A)={ }^{t}\left(\left(\alpha\left({ }^{\sigma} \alpha^{-1}\right)\right)\left({ }^{t} A\right)\right)={ }^{t}\left(c_{\sigma}\left({ }^{t} A\right) c_{\sigma}^{-1}\right)={ }^{t} c_{\sigma}^{-1} A\left({ }^{t} c_{\sigma}\right) .
$$

So the cocycle corresponding to $x^{\mathrm{op}}$ and $\beta$ is precisely the cocycle obtained by precomposing $c$ with the map $A \mapsto{ }^{t} A^{-1}$. This proves the lemma.

Therefore, by the lemma applied to equation (2), $\eta_{\sigma}$ and $\xi_{\sigma}$ correspond to inverse classes in $\operatorname{Br}(L)$, i.e. $y$ is Brauer-equivalent to $x^{\mathrm{op}}$. Since $x$ and $y$ are 9dimensional, they are either both isomorphic to $M_{3}(L)$ or both division algebras. In the first case, $y \cong x^{\text {op }}$ trivially, and in the second case $y$ is Brauer-equivalent to $x^{\mathrm{op}}$, and two division algebras which are Brauer-equivalent are isomorphic. This proves the theorem.

From now on, we will denote by $X^{\text {op }}$ the variety which corresponds to the central simple algebra opposite to the one corresponding to $X ; X^{\mathrm{op}}$ is unique up to isomorphism.

\section{Automorphisms of Severi-Brauer surfaces}

The next result we need is about the action of the automorphism group of a Severi-Brauer surface $X$ on sets of three non-collinear points.

Theorem 3.1. Let $X$ be a 2-dimensional Severi-Brauer variety over a number field $L$, equipped with two $G_{L}$-stable sets $P=\left\{P_{1}, P_{2}, P_{3}\right\}$ and $Q=\left\{Q_{1}, Q_{2}, Q_{3}\right\}$ of non-collinear points. If $\xi: P \rightarrow Q$ is an isomorphism of L-varieties, $\xi$ can be extended to an automorphism $\alpha \in \operatorname{Aut}_{L}(X)$.

Proof. Let $M$ be the smallest Galois extension of $L$ over which the points in $P$ (and $Q$ ) are all individually defined. Let $G_{M / L}=\operatorname{Gal}(M / L)$, as above. Since $X(M) \neq \emptyset$, we have an isomorphism $\phi: X_{M} \rightarrow \mathbb{P}_{M}^{2}$. As before, since Aut $\mathbb{P}_{M}^{2}$ acts transitively on sets of three points in general position, we may assume that $P_{1}, P_{2}, P_{3}$ go to whatever three non-collinear points we want. The following easy lemma provides those points: 
Lemma 3.2. Given a $G_{M / L}$-set $Z$ of order 3, we can find a set $R$ of three non-collinear points in $\mathbb{P}_{M}^{2}$ such that $R$ and $Z$ are isomorphic as $G_{M / L}$-sets.

Proof of lemma. First note that we can immediately find a set of three distinct points $\left\{\alpha_{1}, \alpha_{2}, \alpha_{3}\right\} \subseteq \mathbb{A}^{1}(M)$ which is invariant under $G_{M / L}$ and has the desired structure as a $G_{M / L}$-set. Let $R_{i}=\left(1: \alpha_{i}: \alpha_{i}^{2}\right)$. Then the $R_{i}$ are non-collinear, and the $G_{M / L}$-action on the $R_{i}$ is the same as the action on the $\alpha_{i}$, which is what we wanted.

Applying the lemma with $Z=P$, we obtain a set $R$ of points with the same $G_{M / L^{-a c t i o n}}$ as the one on $P$. So set $\phi\left(P_{i}\right)=R_{i}$ for $i=1,2,3$. We can also construct an isomorphism $\psi: X_{M} \rightarrow \mathbb{P}_{M}^{2}$ such that $\psi\left(Q_{i}\right)=R_{i}$ for $i=1,2,3$. Make cocycles $\eta_{\sigma}=\phi\left({ }^{\sigma} \phi^{-1}\right)$ and $\xi_{\sigma}=\psi\left({ }^{\sigma} \psi^{-1}\right)$. We know that $\eta_{\sigma}$ and $\xi_{\sigma}$ are cohomologous in $H^{1}\left(G_{M / L}, P G L_{3}(M)\right)$, since they both correspond to the same Severi-Brauer variety, and this cohomology group parameterizes Severi-Brauer varieties split by $M$. But in fact, from the construction of $\phi$ and $\psi$ and the fact that $P, Q$, and $R$ have the same $G_{M / L}$-actions, we see that $\eta_{\sigma}$ and $\xi_{\sigma}$ can be viewed as cocycles in $Z^{1}\left(G_{L}, T\right)$, where

$$
T=\left\{A \in P G L_{3}(M): A\left(R_{i}\right)=R_{i} \text { for } i=1,2,3\right\} .
$$

We will need to prove the following

Lemma 3.3. The natural map $i: H^{1}\left(G_{M / L}, T\right) \rightarrow H^{1}\left(G_{M / L}, P G L_{3}(M)\right)$ is injective.

After the lemma is proved, we will conclude that $\eta_{\sigma}$ and $\xi_{\sigma}$ are cohomologous via a coboundary with image in $T$, i.e.

$$
\xi_{\sigma}=B \eta_{\sigma}\left({ }^{\sigma} B^{-1}\right)
$$

with $B \in T$, so that

$$
\begin{aligned}
\psi\left({ }^{\sigma} \psi^{-1}\right) & =B \phi\left({ }^{\sigma} \phi^{-1}\right)\left({ }^{\sigma} B^{-1}\right) \\
\sigma\left(\psi^{-1} B \phi\right) & =\psi^{-1} B \phi
\end{aligned}
$$

for all $\sigma \in G_{L}$. So $\psi^{-1} B \phi$ descends to an $L$-automorphism which extends $\varphi$.

Proof of lemma: First, let $U$ be the set of matrices $B \in G L_{3}(M)$ such that the coordinate vectors in $M^{3}$ representing the $R_{i}$ are eigenvectors of $B$. Then we get the following commutative diagram:

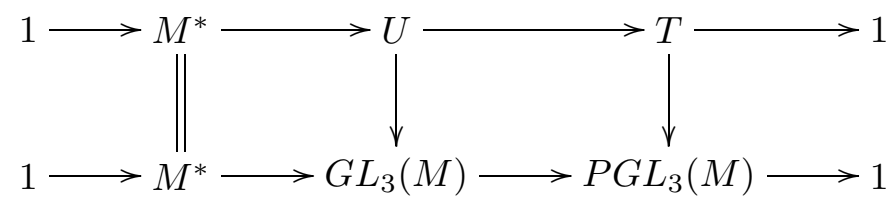

Note that $U$ is abelian (indeed, it is clearly conjugate to the subgroup of $G L_{3}(M)$ consisting of the invertible diagonal matrices). So we can pass to the 
long exact sequence of cohomology associated to this short exact sequence, part of which is

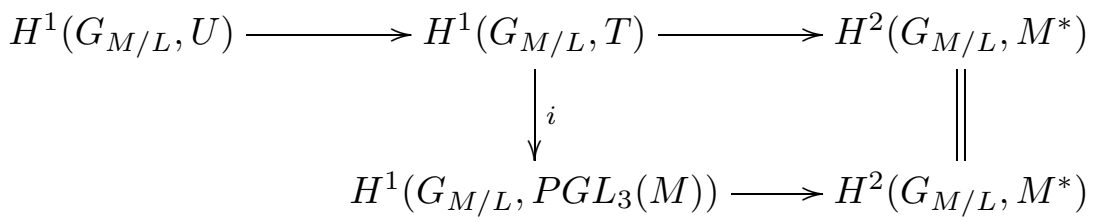

So if we can show that $H^{1}\left(G_{M / L}, U\right)=0$, we'll have that the map at the top of the square is injective, which will imply that $i$ is injective.

First note that $U=C K_{D} C^{-1}$, where $K_{D}$ is the subgroup of diagonal matrices of $G L_{3}(M)$, and $C$ is a change-of-basis matrix. So we have a group isomorphism $U \rightarrow M^{*} \times M^{*} \times M^{*}$ sending $C A C^{-1} \rightarrow\left(A_{11}, A_{22}, A_{33}\right)$.

Let $R=\operatorname{Spec} E$ as an $L$-variety. $E$ is a three-dimensional $L$-algebra, and there are three distinct maps $M \otimes_{L} E \rightarrow M$ corresponding to the three elements of $R(M)$. Then the group homomorphism $M \otimes_{L} E \rightarrow M \times M \times M$ made from these three maps is an isomorphism of rings. Passing to the unit groups of both rings gives a group isomorphism $\left(M \otimes_{L} E\right)^{*} \rightarrow M^{*} \times M^{*} \times M^{*}$. It is easy to check that the composition $U \rightarrow M^{*} \times M^{*} \times M^{*} \rightarrow\left(M \otimes_{L} E\right)^{*}$ actually commutes with the action of $G_{M / L}$ on both sides.

Indeed, another way to see this composition is as the realization of $U$ as the automorphism group of the line bundle over $R_{M}$ corresponding to the invertible sheaf $\mathcal{O}_{R_{M}}(1)$. This automorphism group is isomorphic to $\mathcal{O}_{R_{M}}\left(R_{M}\right)^{*}=\left(M \otimes_{L}\right.$ $E)^{*}$.

But $H^{1}\left(G_{M / L},\left(M \otimes_{L} E\right)^{*}\right)=0$ by an extension of Hilbert's Theorem 90 (see [Ser79], X.1, ex. 2). This proves the lemma.

\section{Reversing the construction}

Now we prove a result about recovering the Del Pezzo surface $D$ from a suitably chosen Severi-Brauer variety $X$.

Theorem 4.1. Let $k$ be a field and let $L / k$ be a quadratic extension with $\operatorname{Gal}(L / k)$ generated by $\sigma$. Suppose $X$ is a Severi-Brauer variety over $L$ such that $X$ and ${ }^{\sigma} X$ correspond to opposite central simple algebras, and suppose we are given a $G_{L}$-stable set of non-collinear points $P:=\left\{P_{1}, P_{2}, P_{3}\right\} \subseteq X(\bar{k})$. Then:

(i) The variety $S$ we constructed in Proposition 2.2 can be descended to a Del Pezzo surface of degree 6 over $k$.

(ii) If we relax the requirements on the above set of data to let $L$ be an étale algebra of degree 2 over $k$, then every Del Pezzo surface of degree 6 over $k$ can be constructed in this way. 
Proof of theorem. In Proposition 2.2 we can take $Y={ }^{\sigma} X$, and by Theorem 3.1 we can assume that the set $Q$ of blown-up points on $Y$ is actually ${ }^{\sigma} P$. So we have blowing-down maps $S \rightarrow X$ and $S \rightarrow{ }^{\sigma} X$ as in the proposition, hence a $\operatorname{map} \varphi: S \rightarrow X \times{ }^{\sigma} X$. Now we prove

Lemma 4.2. $\varphi$ is a closed immersion.

Proof of lemma. It is equivalent to show that $\varphi_{\bar{k}}: S_{\bar{k}} \rightarrow \mathbb{P}_{\bar{k}}^{2} \times \mathbb{P}_{\bar{k}}^{2}$ is a closed immersion. So $\varphi_{\bar{k}}$ is the map which takes $\mathbb{P}^{2}$ blown up at three points and blows down each skew triple of exceptional curves in turn. This description of the map makes it clear that it is injective as a map of sets, and since blowups of projective schemes are projective, $\varphi_{\bar{k}}$ is projective; so $\varphi$ is projective and thus $\varphi$ is a homeomorphism onto its image, a closed subset of $\mathbb{P}^{2} \times \mathbb{P}^{2}$. We now need to check that the map on structure sheaves is surjective, which can be checked on the stalks.

What we need to check is that the map $\mathcal{O}_{\mathbb{P}^{2} \times \mathbb{P}^{2},\left(\pi_{X}(P), \pi_{Y}(P)\right)} \rightarrow \mathcal{O}_{S, P}$ induced by $\varphi$ is surjective for all $P \in S(\bar{k})$. (For convenience, we assume for the remainder of the lemma that everything is over $\bar{k}$ and drop subscripts.) If $P$ lies on at most one of the exceptional lines, then one of the projections $p_{S}: S \rightarrow \mathbb{P}^{2} \times \mathbb{P}^{2} \rightarrow \mathbb{P}^{2}$ restricts to an isomorphism of an open subset of $S$ containing $P$ (namely, $S$ minus a skew triple of exceptional lines not containing $P$ ) onto its image. Thus

$$
\mathcal{O}_{\mathbb{P}^{2}, p_{S}(P)} \rightarrow \mathcal{O}_{\mathbb{P}^{2} \times \mathbb{P}^{2}, \varphi(P)} \rightarrow \mathcal{O}_{S, P}
$$

is surjective, and so the latter map must be as well.

Now suppose $P$ is one of the six points which lies on two exceptional lines. As in [Har77], p. 152, it is enough to check that the map $m_{\mathbb{P}^{2} \times \mathbb{P}^{2}, \varphi(P)} \rightarrow m_{S, P} / m_{S, P}^{2}$ is surjective. Around $P, S$ just looks like the blowup of $\mathbb{A}^{2}$ at a point, and so $m_{S, P} / m_{S, P}^{2}$ is two-dimensional, with generators which cut out the two exceptional lines going through $P$. Each of these two generators comes from exactly one of the maps $m_{\mathbb{P}^{2}, p_{S}(P)} \rightarrow m_{S, P} / m_{S, P}^{2}$ (whichever one does not collapse the line that that generator cuts out). So this implies the surjectivity of the map we want.

Now since $\varphi$ is a closed immersion, it gives an isomorphism of $S$ onto its image, which must be the graph of the birational map $b_{1}: X \rightarrow S \rightarrow{ }^{\sigma} X$ that blows up the $P_{i}$ and then blows down the "other" three lines into the $Q_{i}$. (The graph of a birational map $b$ is the closure of the set of points $\{(a, b(a)) \mid a \in$ domain $(b)\}$.)

The same analysis shows that ${ }^{\sigma} S$ is isomorphic to the graph of the birational map $b_{2}:{ }^{\sigma} X \rightarrow S \rightarrow X$ blowing up the $Q_{i}$ and then blowing down the "other" three lines into the $P_{i}$. Since $b_{1}$ and $b_{2}$ are inverses by construction, the identification of $X \times{ }^{\sigma} X$ with ${ }^{\sigma} X \times X$ by changing the order of the factors induces a map $f_{\sigma}$ from the graph of $b_{1}$ to the graph of $b_{2}$. Then we obtain the following commutative diagram: 


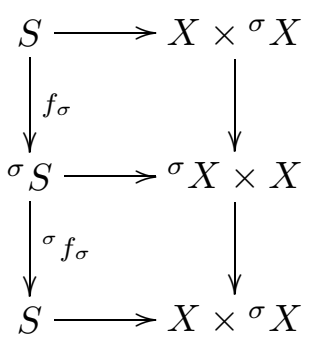

where the maps on the right are the isomorphisms arising from switching the factors. This shows that ${ }^{\sigma} f_{\sigma} \circ f_{\sigma}$ is the identity, since the composition of maps on the right side is the identity. Therefore $f_{\sigma}$ gives descent data for $S$, so that it can be descended (again, as in [Wei56]) to a $k$-variety. This proves statement (i).

As for statement (ii), this merely summarizes what we already know. Given a Del Pezzo surface $D$, if the field given in Proposition 2.1 was quadratic, we can take it to be $L$, and if that field was $k$, we can take $L=k \times k$. Then the Severi-Brauer varieties $X$ and $Y$ associated with $D$ in Proposition 2.1 satisfy $X^{\mathrm{op}}=Y={ }^{\sigma} X$ in either case.

\section{Other results and conclusions}

One remark that should be made about Theorem 4.1 is that the condition ${ }^{\sigma} X=X^{\text {op }}$ is not a very restrictive one. When $L=k \times k$, the varieties $X$ satisfying the condition are generated by starting with any Severi-Brauer surface $X^{\prime} / k$ and then letting $X$ be the disjoint union of $X^{\prime}$ and $\left(X^{\prime}\right)^{\text {op }}$.

When $L$ is a quadratic field extension of $k$, we can view the Galois group generator $\sigma$ as a linear automorphism of $\operatorname{Br} L$. Suppose $x$ is a class of order 3 in $\operatorname{Br} L$. Then $x$ can be written as $2\left(x+{ }^{\sigma} x\right)+2\left(x-{ }^{\sigma} x\right)$, so

$$
(\operatorname{Br} L)[3]=(\operatorname{Br} L)^{G_{L / k}}[3] \oplus W,
$$

where $W$ is the set of classes of algebras corresponding to varieties $X$ satisfying the condition ${ }^{\sigma} X=X^{\mathrm{op}}$.

In fact, a little more can be said: the spectral sequence

$$
E_{2}^{p, q}:=H^{p}\left(G_{L / k}, H^{q}\left(G_{L}, \bar{L}^{*}\right)\right) \Rightarrow H^{p+q}\left(G_{k}, \bar{k}^{*}\right)
$$

yields the usual exact sequence

$$
0 \rightarrow E_{2}^{2,0} \rightarrow \operatorname{Br} k \rightarrow E_{2}^{0,2} \rightarrow E_{2}^{3,0},
$$

but $E_{2}^{0,2}=(\operatorname{Br} L)^{G_{L / k}}$ and $E_{2}^{3,0}=E_{2}^{1,0}=0$ by Hilbert's Theorem 90 and the fact that $L / k$ is cyclic. Since multiplication-by-3 is the identity on the 2-torsion group $E_{2}^{2,0}$, the natural map

$$
(\mathrm{Br} k)[3] \rightarrow(\operatorname{Br} L)^{G_{L / k}}[3]
$$

is an isomorphism, so that $(\operatorname{Br} L)[3] \cong(\operatorname{Br} k)[3] \oplus W$. 
Finally, we simultaneously sum up the results we have established and include the proof of one last remark:

Theorem 5.1. Giving a Del Pezzo surface of degree 6 over a field $k$ of characteristic zero is equivalent to giving the following data:

1. an étale algebra $L$ of degree 2 over $k$

2. a Severi-Brauer variety $X$ of dimension 2 over $L$ such that ${ }^{\sigma} X=X^{\mathrm{op}}$, where $\sigma$ generates $\operatorname{Gal}(L / k)$

3. a subscheme $P$ of $X$ consisting of three geometric non-collinear points

Moreover, two Del Pezzo surfaces $S_{i}$ corresponding to $L_{i}, X_{i}$, and $P_{i}(i=1,2)$ are isomorphic if and only if $L_{1} \cong L_{2}$ and there is an isomorphism $X_{1} \rightarrow X_{2}$ such that $P_{1}$ maps isomorphically onto $P_{2}$.

Proof of theorem. All we need to check is the last statement. For the "if" direction, this simply follows from the description of $S_{i}$ given in the proof of Theorem 4.1, as the graph of a birational map constructed in terms of $X_{i},{ }^{\sigma} X_{i}$, and $P_{i}$. For the "only if" direction, note that we constructed the objects $L_{i}$, $X_{i}$, and $P_{i}$ intrinsically from $S_{i}$. If $S_{1}$ and $S_{2}$ are isomorphic, we naturally get the isomorphisms given in the statement of the theorem. (The only choice we made was between $X$ and ${ }^{\sigma} X$, but these varieties are isomorphic over $L$, and, as noted before, Theorem 3.1 implies that we can make the isomorphism send $P$ to ${ }^{\sigma} P$.)

\section{Acknowledgements}

I thank my advisor Bjorn Poonen, who posed and helped answer many of the questions raised by this paper.

\section{References}

[Har77] R. Hartshorne, Algebraic geometry, Springer-Verlag, New York, 1977, Graduate Texts in Mathematics, No. 52.

[Man74] Y. I. Manin, Cubic Forms. North-Holland, Amsterdam, 1974,

[Ser79] J.-P. Serre, Local fields, Springer-Verlag, New York, 1979,

[Wei56] A. Weil, The field of definition of a variety, American Journal of Mathematics, 78 (1956), 509-524.

Department of Mathematics, University of California, Berkeley, CA 94720-3840, USA

E-mail address: corn@math.berkeley.edu

$U R L:$ http://math. berkeley.edu/ corn 